\title{
Weaving of $K$-g-frames in Hilbert spaces
}

\author{
Xiang-Chun Xiao ${ }^{\mathrm{a}, *}$, Guo-Rong Zhou ${ }^{\mathrm{a}}$, Yu-Can $\mathrm{Zhu}^{\mathrm{b}}$ \\ a Department of Mathematics, Xiamen University of Technology, Xiamen 361024 China \\ b Department of Mathematics and Computer Science, Fuzhou University, Fuzhou 350116 China
}

*Corresponding author, e-mail: xxc570@163.com

Received 10 Jan 2019

Accepted 12 May 2019

\begin{abstract}
In this study, we mainly discuss the weaving of $K$-g-frames in Hilbert spaces. Note that the concept of weaving was recently proposed by Bemrose et al to solve a question in distributed signal processing. We give a sufficient condition such that the left sequence can still be $K$-woven in $R(K)$ by deleting some elements from a $K$-woven pair of $K$-g-frames. We then give three different types of perturbation conditions such that, under them, the $K$-g-frames $\left\{\Lambda_{j}: j \in J\right\}$ and $\left\{\Gamma_{j}: j \in J\right\}$ or the types $\left\{\Lambda_{j} T_{1}^{*}: j \in J\right\}$ and $\left\{\Gamma_{j} T_{2}^{*}: j \in J\right\}$ can be $K$-woven in $R(K)$, where $T_{1}, T_{2}$ are surjective operators on $\mathscr{U}$.
\end{abstract}

KEYWORDS: G-frame, $K$-g-frame, weaving, perturbation

MSC2010: 42C15

\section{INTRODUCTION}

Sun ${ }^{1}$ proposed a more general type of frame called $g$-frame to deal with all existing frames at that time as a united object. Given $J$ being a countable index set; $\mathscr{U}, \mathscr{V}_{j}, j \in J$, being Hilbert spaces; and $\Lambda_{j}$ being a bounded linear operator from $\mathscr{U}$ to $\mathscr{V}_{j}$, recall that $\left\{\Lambda_{j}: j \in J\right\}$ is called a $g$-frame for $\mathscr{U}$ with respect to $\left\{\mathscr{V}_{j}: j \in J\right\}$ if there exist $A, B>0$ such that

$$
A\|f\|^{2} \leqslant \sum_{j \in J}\left\|\Lambda_{j} f\right\|^{2} \leqslant B\|f\|^{2}, \quad \forall f \in \mathscr{U} .
$$

Here, $A$ and $B$ are the lower and upper frame bounds, respectively, for the g-frame $\left\{\Lambda_{j}: j \in J\right\}$. Xiao et $\mathrm{al}^{2}$ further generalized $g$-frames ${ }^{3-5}$ and $K$ frames ${ }^{6-8}$ and introduced another notion, namely, $K$-g-frames. For more information on $g$-frames and $K$-g-frames, see Refs. 9-11 and the references therein.

Note that the notion of weaving was recently proposed by Bemrose et al ${ }^{12-14}$ to simulate a question in distributed signal processing. Since then weaving as a research hotspot has been studied by many scholars. We refer the readers to check Refs. 15-18 for more information on the weaving of $g$-frames or fusion frames and Ref. 19 for information on the weaving of $K$-frames.

In this study, we will mainly discuss the erasures and perturbations of weaving for $K$-g-frames in Hilbert spaces. We give a sufficient condition such that the left sequence can still be $K$-woven in $R(K)$ by deleting some elements from a $K$-woven pair of $K$-g-frames. We then give three different types of perturbation conditions such that, under them, the $K$-g-frames $\left\{\Lambda_{j}: j \in J\right\}$ and $\left\{\Gamma_{j}: j \in J\right\}$ or the types $\left\{\Lambda_{j} T_{1}^{*}: j \in J\right\}$ and $\left\{\Gamma_{j} T_{2}^{*}: j \in J\right\}$ can be $K$-woven in $R(K)$, where $T_{1}$ and $T_{2}$ are surjective operators on $\mathscr{U}$.

Throughout this study, we will adopt the following notations: $\mathscr{H}$ is a separable Hilbert space; $I_{\mathscr{H}}$ is the identity operator for $\mathscr{H} ; L(X, Y)$ is the collection of all bounded linear operators from $X$ to $Y$, where $X, Y$ are Banach spaces, and if $X=Y$, then $L(X, Y)$ is denoted by $L(X)$; the range and the kernel of $K \in$ $L(\mathscr{H})$ are denoted by $R(K)$ and $N(K)$, respectively; finally, the pseudo-inverse of $K \in L(\mathscr{H})$ is denoted by $K^{\dagger}$.

\section{PRELIMINARIES OF $K$-G-FRAMES}

In this section, we recall the definitions and some basic properties of $K$-g-frames and weaving, and apply the woven principle to $K$-g-frames.

Definition 1 [Ref. 2] A sequence $\left\{\Lambda_{j} \in L\left(\mathscr{U}, \mathscr{V}_{j}\right)\right.$ : $j \in J\}$ is called a $K$-g-frame for $\mathscr{U}$ with respect to $\left\{\mathscr{V}_{j}: j \in J\right\}$ if there exist $A, B>0$ such that

$$
A\left\|K^{*} f\right\|^{2} \leqslant \sum_{j \in J}\left\|\Lambda_{j} f\right\|^{2} \leqslant B\|f\|^{2}, \quad \forall f \in \mathscr{U} .
$$

We call $A$ and $B$ the lower frame bound and the upper frame bound, respectively, for the $K$-g-frame 
$\left\{\Lambda_{j}: j \in J\right\}$. We call $\left\{\Lambda_{j}: j \in J\right\}$ the g-Bessel sequence if only the right-hand side of (1) holds.

If $A\left\|K^{*} f\right\|^{2}=\sum_{j \in J}\left\|\Lambda_{j} f\right\|^{2}, \forall f \in \mathscr{U}$, we call $\left\{\Lambda_{j}: j \in J\right\}$ a tight $K$-g-frame; furthermore, if $A=1$, $\left\{\Lambda_{j}: j \in J\right\}$ is called a Parseval $K$-g-frame.

Observe that, from (1), $\left\{\Lambda_{j}: j \in J\right\}$ is an $I_{\mathscr{U}^{-}}$-gframe for $\mathscr{U}$ if and only if $\left\{\Lambda_{j}: j \in J\right\}$ is a $g$-frame for $\mathscr{U}$.

The concept of weaving of frames was introduced by Bemrose et al ${ }^{12}$ to simulate a question in distributed signal processing. We now recall it as follows.

Definition 2 [Ref. 12] Let $\left\{f_{i}\right\}_{i \in I}$ and $\left\{g_{i}\right\}_{i \in I}$ be frames for $\mathscr{H}$. If for any partition $\left\{\sigma_{j}\right\}_{j=1}^{2}$ of $I$ there exist $A, B>0$ such that $\left\{f_{i}\right\}_{i \in \sigma_{1}} \cup\left\{g_{i}\right\}_{i \in \sigma_{2}}$ is a frame for $\mathscr{H}$ with the frame bounds $A$ and $B$, then we consider that $\left\{f_{i}\right\}_{i \in I}$ and $\left\{g_{i}\right\}_{i \in I}$ are woven in $\mathscr{H}$ with the frame bounds $A$ and $B$, and $\left\{f_{i}\right\}_{i \in \sigma_{1}} \cup$ $\left\{g_{i}\right\}_{i \in \sigma_{2}}$ is called a weaving.

In this study, we will apply the woven principle to $K$-g-frames.

Definition 3 Let $\left\{\Lambda_{j}: j \in J\right\}$ and $\left\{\Gamma_{j}: j \in J\right\}$ be $K$ g-frames for $\mathscr{U}$ with respect to $\left\{\mathscr{V}_{j}: j \in J\right\}$. If for any partition $\left\{\sigma_{j}\right\}_{j=1}^{2}$ of $J$ there exist $A, B>0$ such that $\left\{\Lambda_{j}\right\}_{i \in \sigma_{1}} \cup\left\{\Gamma_{j}\right\}_{i \in \sigma_{2}}$ is a $K$-g-frame for $\mathscr{U}$ with the frame bounds $A$ and $B$, then we consider that $\left\{\Lambda_{j}\right.$ : $j \in J\}$ and $\left\{\Gamma_{j}: j \in J\right\}$ are $K$-woven in $\mathscr{U}$ with the frame bounds $A$ and $B$, and each $\left\{\Lambda_{j}\right\}_{i \in \sigma_{1}} \cup\left\{\Gamma_{j}\right\}_{i \in \sigma_{2}}$ is called a weaving.

Note that, if $K=I_{\mathscr{H}}$, then $K$-frames are the classical frames for $\mathscr{H}$ and, at the same time, the weaving of the $K$-frames is the weaving of the classical frames.

Assume that $\left\{\Lambda_{j} \in L\left(\mathscr{U}, \mathscr{V}_{j}\right): j \in J\right\}$ is a $\mathrm{g}$ Bessel sequence in $\mathscr{U}$; then, the synthesis operator $T$, analysis operator $U$, and frame operator $S$ of $\left\{\Lambda_{j}: j \in J\right\}$ are defined as follows:

$$
\begin{gathered}
T: l^{2}\left(\left\{\mathscr{V}_{j}\right\}_{j \in J}\right) \rightarrow \mathscr{U}, \quad T\left(\left\{g_{j}\right\}_{j \in J}\right)=\sum_{j \in J} \Lambda_{j}^{*} g_{j}, \\
U: \mathscr{U} \rightarrow l^{2}\left(\left\{\mathscr{V}_{j}\right\}_{j \in J}\right), \quad U f=\left\{\Lambda_{j} f\right\}_{j \in J}, \\
S: \mathscr{U} \rightarrow \mathscr{U}, \quad S f=\sum_{j \in J} \Lambda_{j}^{*} \Lambda_{j} f,
\end{gathered}
$$

where $l^{2}\left(\left\{\mathscr{V}_{j}\right\}_{j \in J}\right)$ is a Hilbert space defined as

$$
l^{2}\left(\left\{\mathscr{V}_{j}\right\}_{j \in J}\right)=\left\{\left\{g_{j}\right\}_{j \in J}: g_{j} \in \mathscr{V}_{j}, j \in J, \sum_{j \in J}\left\|g_{j}\right\|^{2}<\infty\right\}
$$

with inner product $\left\langle\left\{f_{j}\right\}_{j \in J},\left\{g_{j}\right\}_{j \in J}\right\rangle=\sum_{j \in J}\left\langle f_{j}, g_{j}\right\rangle$. It is easy to check that $U=T^{*}$ and $S=T U$.
Lemma 1 (Ref. 20) Suppose that $\mathscr{H}_{1}$ and $\mathscr{H}_{2}$ are two Hilbert spaces and that $Q \in L\left(\mathscr{H}_{1}, \mathscr{H}_{2}\right)$ is an operator with a closed range. Then, there exists a unique bounded operator $Q^{\dagger}: \mathscr{H}_{2} \rightarrow \mathscr{H}_{1}$, called the pseudo-inverse operator of $Q$, satisfying

$$
\begin{aligned}
& N\left(Q^{\dagger}\right)=R(Q)^{\perp}, \quad R\left(Q^{\dagger}\right)=N(Q)^{\perp}, \\
& Q Q^{\dagger}=P_{R(Q)}, \quad Q^{\dagger} Q=P_{R\left(Q^{\dagger}\right)} .
\end{aligned}
$$

If $Q$ is bounded and invertible, then $Q^{\dagger}=Q^{-1}$.

In this study, we always assume that $K \in L(\mathscr{U})$ is an operator with a closed range. By Lemma 1, there exists a pseudo-inverse operator $K^{\dagger}$ such that $K K^{\dagger}=P_{R(K)}$. It follows that $I_{R(K)}=I_{R(K)}^{*}=\left(K^{\dagger}\right)^{*} K^{*}$. Hence, for any $f \in R(K)$,

$$
\|f\|=\left\|\left(K^{\dagger}\right)^{*} K^{*} f\right\| \leqslant\left\|\left(K^{\dagger}\right)^{*}\right\|\left\|K^{*} f\right\|=\left\|K^{\dagger}\right\|\left\|K^{*} f\right\| .
$$

\section{ERASURES OF THE WEAVING OF $K$-G-FRAMES}

In this section, we provide a sufficient condition such that the left sequence can still be $K$-woven in $R(K)$ by deleting some elements from a $K$-woven pair of $K$-g-frames.

Theorem 1 Let $K \in L(\mathscr{U})$ be an operator with a closed range. Suppose that $\left\{\Lambda_{j}: j \in J\right\}$ and $\left\{\Gamma_{j}: j \in J\right\}$ are $K$-g-frames for $\mathscr{U}$ with respect to $\left\{\mathscr{V}_{j}: j \in J\right\}$ and are $K$-woven in $\mathscr{U}$ with the universal bounds $A$ and $B$. If there exist $\sigma \subset J$ and $\alpha, \beta, \gamma \geqslant 0$ satisfying $A>\beta+(\alpha B+\gamma)\left\|K^{\dagger}\right\|^{2}$ such that

$$
\sum_{j \in \sigma}\left\|\Lambda_{j} f\right\|^{2} \leqslant \alpha \sum_{j \in \sigma}\left\|\Gamma_{j} f\right\|^{2}+\beta\left\|K^{*} f\right\|^{2}+\gamma\|f\|^{2}
$$

for all $f \in \mathscr{U}$, then $\left\{\Lambda_{j}: j \in J \backslash \sigma\right\}$ and $\left\{\Gamma_{j}: j \in J \backslash \sigma\right\}$ are $K$-woven in $R(K)$ with the universal bounds $A-$ $\beta-(\alpha B+\gamma)\left\|K^{\dagger}\right\|^{2}$ and $B$.

Proof: For any partition $\left\{\sigma_{j}\right\}_{j=1}^{2}$ of $J \backslash \sigma$, and any $f \in R(K) \subset \mathscr{U}$, we have

$$
\begin{aligned}
& \sum_{j \in \sigma_{1}}\left\|\Lambda_{j} f\right\|^{2}+\sum_{j \in \sigma_{2}}\left\|\Gamma_{j} f\right\|^{2} \\
& =\left(\sum_{j \in J \backslash \sigma_{2}}\left\|\Lambda_{j} f\right\|^{2}+\sum_{j \in \sigma_{2}}\left\|\Gamma_{j} f\right\|^{2}\right)-\sum_{j \in \sigma}\left\|\Lambda_{j} f\right\|^{2} \\
& \geqslant A\left\|K^{*} f\right\|^{2}-\left(\alpha \sum_{j \in \sigma}\left\|\Gamma_{j} f\right\|^{2}+\beta\left\|K^{*} f\right\|^{2}+\gamma\|f\|^{2}\right) \\
& \geqslant(A-\beta)\left\|K^{*} f\right\|^{2}-(\alpha B+\gamma)\|f\|^{2} \\
& \geqslant(A-\beta)\left\|K^{*} f\right\|^{2}-(\alpha B+\gamma)\left\|K^{\dagger}\right\|^{2}\left\|K^{*} f\right\|^{2} \\
& =\left[A-\beta-(\alpha B+\gamma)\left\|K^{\dagger}\right\|^{2}\right]\left\|K^{*} f\right\|^{2},
\end{aligned}
$$

where the first inequality is deduced by (7) and that $\left\{\Lambda_{j}: j \in J\right\}$ and $\left\{\Gamma_{j}: j \in J\right\}$ are $K$-woven with the 
universal bounds $A$ and $B$, and the third inequality is deduced by (6). On the other hand, we have

$$
\begin{aligned}
\sum_{j \in \sigma_{1}}\left\|\Lambda_{j} f\right\|^{2} & +\sum_{j \in \sigma_{2}}\left\|\Gamma_{j} f\right\|^{2} \\
& \leqslant \sum_{j \in \sigma_{1} \cup \sigma}\left\|\Lambda_{j} f\right\|^{2}+\sum_{j \in \sigma_{2}}\left\|\Gamma_{j} f\right\|^{2} \leqslant B\|f\|^{2} .
\end{aligned}
$$

We can also know that $\left\{\Lambda_{j}: j \in J \backslash \sigma\right\}$ and $\left\{\Gamma_{j}: j \in\right.$ $J \backslash \sigma\}$ are $K$-g-frames for $R(K)$ if we take $\sigma_{1}=J \backslash \sigma$ and $\varnothing$ from (8). Hence $\left\{\Lambda_{j}: j \in J \backslash \sigma\right\}$ and $\left\{\Gamma_{j}: j \in\right.$ $J \backslash \sigma\}$ are $K$-woven in $R(K)$.

We can easily obtain the following result if we take $K=I_{\mathscr{U}}$ in Theorem 1 .

Corollary 1 Suppose that $\left\{\Lambda_{j}: j \in J\right\}$ and $\left\{\Gamma_{j}: j \in J\right\}$ are $g$-frames for $\mathscr{U}$ with respect to $\left\{\mathscr{V}_{j}: j \in J\right\}$ and are woven in $\mathscr{U}$ with the universal frame bounds $A$ and $B$. If there exist $\sigma \subset J$ and $\alpha \beta \geqslant 0$ satisfying $A>\alpha B+\beta$ such that

$$
\sum_{j \in \sigma}\left\|\Lambda_{j} f\right\|^{2} \leqslant \alpha \sum_{j \in \sigma}\left\|\Gamma_{j} f\right\|^{2}+\beta\|f\|^{2}, \quad \forall f \in \mathscr{U},
$$

then $\left\{\Lambda_{j}: j \in J \backslash \sigma\right\}$ and $\left\{\Gamma_{j}: j \in J \backslash \sigma\right\}$ are woven in $\mathscr{U}$ with the universal frame bounds $A-\alpha B-\beta$ and $B$.

\section{PERTURBATIONS OF THE WEAVING OF $K$-G-FRAMES}

In this section, we mainly discuss the perturbation stabilities of the weaving of $\left\{\Lambda_{j}: j \in J\right\}$ and $\left\{\Gamma_{j}: j \in\right.$ $J\}$ under different types of perturbation conditions.

Given that $\left\{\Lambda_{j}: j \in J\right\}$ and $\left\{\Gamma_{j}: j \in J\right\}$ are $K$-gframes for $\mathscr{U}$, we show that, under condition (10), $\left\{\Lambda_{j} T_{1}^{*}: j \in J\right\}$ and $\left\{\Gamma_{j} T_{2}^{*}: j \in J\right\}$ are $K$-woven in $R(K)$ for some surjective operators $T_{1}, T_{2}$ on $\mathscr{U}$.

Theorem 2 Let $K \in L(\mathscr{U})$ be an operator with a closed range. Suppose that $\left\{\Lambda_{j}: j \in J\right\}$ and $\left\{\Gamma_{j}: j \in J\right\}$ are $K$-g-frames for $\mathscr{U}$ with respect to $\left\{\mathscr{V}_{j}: j \in J\right\}$ with the frame bounds $A_{1}, B_{1}$ and $A_{2}, B_{2}$, respectively. Suppose that $T_{1}, T_{2} \in L(\mathscr{U})$ are surjective on $\mathscr{U}$ and satisfy $T_{i} K=K T_{i}, i=1$, 2. If there exist $\alpha, \beta, \gamma \geqslant 0$ satisfying $\frac{\sqrt{A_{1}}}{\left\|T_{1}^{\dagger}\right\|}-\frac{\gamma}{\| T_{2}^{i \Uparrow \|}}>\left(\sqrt{B_{1}}\left\|T_{2}-T_{1}\right\|+\alpha \sqrt{B_{1}}\left\|T_{2}\right\|+\right.$ $\left.\beta \sqrt{B_{2}}\left\|T_{2}\right\|\right)\left\|K^{\dagger}\right\|$ such that, $\forall f \in \mathscr{U}$,

$$
\begin{aligned}
\left(\sum_{j \in J}\left\|\left(\Gamma_{j}-\Lambda_{j}\right) f\right\|^{2}\right)^{1 / 2} \leqslant \alpha\left(\sum_{j \in J}\left\|\Lambda_{j} f\right\|^{2}\right)^{1 / 2} & \\
& +\beta\left(\sum_{j \in J}\left\|\Gamma_{j} f\right\|^{2}\right)^{1 / 2}+\gamma\left\|K^{*} f\right\|,
\end{aligned}
$$

then $\left\{\Lambda_{j} T_{1}^{*}: j \in J\right\}$ and $\left\{\Gamma_{j} T_{2}^{*}: j \in J\right\}$ are $K$-woven in $R(K)$ with the universal frame bounds $\left(\frac{\sqrt{A_{1}}}{\left\|T_{1}^{i}\right\|}-\frac{\gamma}{\left\|T_{2}^{i}\right\|}-\right.$
$\left.\left(\sqrt{B_{1}}\left\|T_{2}-T_{1}\right\|+\alpha \sqrt{B_{1}}\left\|T_{2}\right\|+\beta \sqrt{B_{2}}\left\|T_{2}\right\|\right)\left\|K^{\dagger}\right\|\right)^{2}$ and $B_{1}\left\|T_{1}\right\|^{2}+B_{2}\left\|T_{2}\right\|^{2}$.

Proof: Since $T_{1} \in L(\mathscr{U})$ is surjective on $\mathscr{U}$, similar to (6), we obtain, for any $f \in \mathscr{U}$,

$$
\|f\|=\left\|\left(T_{1}^{\dagger}\right)^{*} T_{1}^{*} f\right\| \leqslant\left\|T_{1}^{\dagger}\right\|\left\|T_{1}^{*} f\right\| .
$$

Hence we obtain

$$
\left\|T_{1}^{*} f\right\| \geqslant \frac{1}{\left\|T_{1}^{\dagger}\right\|}\|f\|, \quad \forall f \in \mathscr{U} .
$$

We can now show that $\left\{\Lambda_{j} T_{1}^{*}: j \in J\right\}$ and $\left\{\Gamma_{j} T_{2}^{*}\right.$ : $j \in J\}$ are $K$-g-frames for $\mathscr{U}$. Since $\left\{\Lambda_{j}: j \in J\right\}$ is a $K$-g-frame for $\mathscr{U}$, therefore (1) holds. For any $f \in$ $\mathscr{U}$, we obtain

$$
\begin{aligned}
\frac{A_{1}}{\left\|T_{1}^{\dagger}\right\|^{2}}\left\|K^{*} f\right\|^{2} & \leqslant A_{1}\left\|T_{1}^{*} K^{*} f\right\|^{2}=A_{1}\left\|K^{*} T_{1}^{*} f\right\|^{2} \\
& \leqslant \sum_{j \in J}\left\|\Lambda_{j} T_{1}^{*} f\right\|^{2} \\
& \leqslant B_{1}\left\|T_{1}^{*} f\right\|^{2} \leqslant B_{1}\left\|T_{1}\right\|^{2}\|f\|^{2}
\end{aligned}
$$

where the first inequality is deduced by (12). Hence $\left\{\Lambda_{j} T_{1}^{*}: j \in J\right\}$ is a $K$-g-frame for $\mathscr{U}$. Similarly, we can show that $\left\{\Gamma_{j} T_{2}^{*}: j \in J\right\}$ is a $K$-g-frame for $\mathscr{U}$.

Next, we show that $\left\{\Lambda_{j} T_{1}^{*}: j \in J\right\}$ and $\left\{\Gamma_{j} T_{2}^{*}\right.$ : $j \in J\}$ are woven in $R(K)$. For any partition $\left\{\sigma_{j}\right\}_{j=1}^{2}$ of $J$, and any $f \in \mathscr{U}$, we have

$$
\begin{aligned}
\sum_{j \in \sigma_{1}}\left\|\Lambda_{j} T_{1}^{*} f\right\|^{2}+\sum_{j \in \sigma_{2}}\left\|\Gamma_{j} T_{2}^{*} f\right\|^{2} & \\
& \leqslant\left(B_{1}\left\|T_{1}\right\|^{2}+B_{2}\left\|T_{2}\right\|^{2}\right)\|f\|^{2} .
\end{aligned}
$$

Let $x=\left\{\Lambda_{j} T_{1}^{*} f\right\}_{j \in \sigma_{1}} \cup\left\{\Lambda_{j} T_{1}^{*} f\right\}_{j \in \sigma_{2}}$ and $y=$ $\{0\}_{j \in \sigma_{1}} \cup\left\{\Lambda_{j}\left(T_{2}-T_{1}\right)^{*} f+\left(\Gamma_{j}-\Lambda_{j}\right) T_{2}^{*} f\right\}_{j \in \sigma_{2}}$. Then $x, y \in l^{2}\left(\left\{\mathscr{V}_{j}\right\}_{j \in J}\right)$ since $\left\{\Lambda_{j} T_{1}^{*}: j \in J\right\}$ and $\left\{\Lambda_{j}\left(T_{2}-\right.\right.$ $\left.\left.T_{1}\right)^{*}: j \in J\right\} \bigcup\left\{\left(\Lambda_{j}-\Gamma_{j}\right) T_{2}^{*}: j \in J\right\}$ can be proved to be $\mathrm{g}$-Bessel sequences in $\mathscr{U}$. Furthermore, there is

$$
x+y=\left\{\Lambda_{j} T_{1}^{*} f\right\}_{j \in \sigma_{1}} \bigcup\left\{\Gamma_{j} T_{2}^{*} f\right\}_{j \in \sigma_{2}} \in l^{2}\left(\left\{\mathscr{V}_{j}\right\}_{j \in J}\right) .
$$


Hence for any $f \in R(K)$, we obtain

$$
\begin{aligned}
& \left(\sum_{j \in \sigma_{1}}\left\|\Lambda_{j} T_{1}^{*} f\right\|^{2}+\sum_{j \in \sigma_{2}}\left\|\Gamma_{j} T_{2}^{*} f\right\|^{2}\right)^{1 / 2} \\
& =\|x+y\| \geqslant\|x\|-\|y\| \\
& =\left(\sum_{j \in J}\left\|\Lambda_{j} T_{1}^{*} f\right\|^{2}\right)^{1 / 2} \\
& -\left(\sum_{j \in \sigma_{2}}\left\|\Lambda_{j}\left(T_{2}-T_{1}\right)^{*} f+\left(\Gamma_{j}-\Lambda_{j}\right) T_{2}^{*} f\right\|^{2}\right)^{1 / 2} \\
& \geqslant \sqrt{A_{1}}\left\|K^{*} T_{1}^{*} f\right\|-\left(\sum_{j \in \sigma_{2}}\left\|\Lambda_{j}\left(T_{2}-T_{1}\right)^{*} f\right\|^{2}\right)^{1 / 2} \\
& -\left(\sum_{j \in \sigma_{2}}\left\|\left(\Gamma_{j}-\Lambda_{j}\right) T_{2}^{*} f\right\|^{2}\right)^{1 / 2} \\
& \geqslant \sqrt{A_{1}}\left\|K^{*} T_{1}^{*} f\right\|-\left(\sum_{j \in J}\left\|\Lambda_{j}\left(T_{2}-T_{1}\right)^{*} f\right\|^{2}\right)^{1 / 2} \\
& -\left(\sum_{j \in J}\left\|\left(\Gamma_{j}-\Lambda_{j}\right) T_{2}^{*} f\right\|^{2}\right)^{1 / 2} \\
& \geqslant \sqrt{A_{1}}\left\|T_{1}^{*} K^{*} f\right\|-\sqrt{B_{1}}\left\|\left(T_{2}-T_{1}\right)^{*} f\right\|-\gamma\left\|K^{*} T_{2}^{*} f\right\| \\
& -\alpha\left(\sum_{j \in J}\left\|\Lambda_{j} T_{2}^{*} f\right\|^{2}\right)^{1 / 2}-\beta\left(\sum_{j \in J}\left\|\Gamma_{j} T_{2}^{*} f\right\|^{2}\right)^{1 / 2} \\
& \geqslant \sqrt{A_{1}}\left\|T_{1}^{*} K^{*} f\right\|-\sqrt{B_{1}}\left\|T_{2}-T_{1}\right\|\|f\|-\gamma\left\|T_{2}^{*} K^{*} f\right\| \\
& -\alpha \sqrt{B_{1}}\left\|T_{2}^{*} f\right\|-\beta \sqrt{B_{2}}\left\|T_{2}^{*} f\right\| \\
& \geqslant\left(\frac{\sqrt{A_{1}}}{\left\|T_{1}^{\dagger}\right\|}-\frac{\gamma}{\left\|T_{2}^{\dagger}\right\|}\right)\left\|K^{*} f\right\|-\left(\sqrt{B_{1}}\left\|T_{2}-T_{1}\right\|\right. \\
& \left.+\alpha \sqrt{B_{1}}\left\|T_{2}\right\|+\beta \sqrt{B_{2}}\left\|T_{2}\right\|\right)\|f\| \\
& \geqslant\left(\frac{\sqrt{A_{1}}}{\left\|T_{1}^{\dagger}\right\|}-\frac{\gamma}{\left\|T_{2}^{\dagger}\right\|}\right)\left\|K^{*} f\right\|-\left(\sqrt{B_{1}}\left\|T_{2}-T_{1}\right\|\right. \\
& \left.+\alpha \sqrt{B_{1}}\left\|T_{2}\right\|+\beta \sqrt{B_{2}}\left\|T_{2}\right\|\right)\left\|K^{\dagger}\right\|\left\|K^{*} f\right\| \\
& =\left(\frac{\sqrt{A_{1}}}{\left\|T_{1}^{\dagger}\right\|}-\frac{\gamma}{\left\|T_{2}^{\dagger}\right\|}-\left(\sqrt{B_{1}}\left\|T_{2}-T_{1}\right\|\right.\right. \\
& \left.\left.+\alpha \sqrt{B_{1}}\left\|T_{2}\right\|+\beta \sqrt{B_{2}}\left\|T_{2}\right\|\right)\left\|K^{\dagger}\right\|\right)\left\|K^{*} f\right\|,
\end{aligned}
$$

where the second inequality is obtained by Minkowski's inequality, the sixth inequality by (12), and the seventh inequality by (6). Hence $\left\{\Lambda_{j} T_{1}^{*}: j \in J\right\}$ and $\left\{\Gamma_{j} T_{2}^{*}: j \in J\right\}$ are $K$-woven in $R(K)$.

If $T_{1}=T_{2}$ in Theorem 2 , we can easily have the following corollary.

Corollary 2 Let $K \in L(\mathscr{U})$ be an operator with a closed range. Suppose that $\left\{\Lambda_{j}: j \in J\right\}$ and $\left\{\Gamma_{j}\right.$ : $j \in J\}$ are $K$-g-frames for $\mathscr{U}$ with respect to $\left\{\mathscr{V}_{j}\right.$ : $j \in J\}$ with the frame bounds $A_{1}, B_{1}$ and $A_{2}, B_{2}$, respectively. Suppose that $T \in L(\mathscr{U})$ is surjective on $\mathscr{U}$ and satisfies $T K=K T$. If there exist $\alpha, \beta, \gamma \geqslant 0$ satisfying $\sqrt{A_{1}}-\gamma>\left(\alpha \sqrt{B_{1}}+\beta \sqrt{B_{2}}\right)\|T\|\left\|T^{\dagger}\right\|\left\|K^{\dagger}\right\|$ such that, $\forall f \in \mathscr{U}$,

$$
\begin{aligned}
\left(\sum_{j \in J}\left\|\left(\Gamma_{j}-\Lambda_{j}\right) f\right\|^{2}\right)^{1 / 2} & \leqslant \alpha\left(\sum_{j \in J}\left\|\Lambda_{j} f\right\|^{2}\right)^{1 / 2} \\
& +\beta\left(\sum_{j \in J}\left\|\Gamma_{j} f\right\|^{2}\right)^{1 / 2}+\gamma\left\|K^{*} f\right\|,
\end{aligned}
$$

then $\left\{\Lambda_{j} T^{*}: j \in J\right\}$ and $\left\{\Gamma_{j} T^{*}: j \in J\right\}$ are $K$ woven in $R(K)$ with the universal frame bounds $\left(\left(\sqrt{A_{1}}-\gamma\right) /\left\|T^{\dagger}\right\|-\left(\alpha \sqrt{B_{1}}+\beta \sqrt{B_{2}}\right)\|T\|\left\|K^{\dagger}\right\|\right)^{2}$ and $\left(B_{1}+B_{2}\right)\|T\|^{2}$.

If $\alpha=\beta=\gamma=0$ in Theorem 2, then from (10) we can deduce that $\Lambda_{j}=\Gamma_{j}, \forall j \in J$, and a result follows from Theorem 2 .

Corollary 3 Let $K \in L(\mathscr{U})$ be an operator with a closed range. Suppose that $\left\{\Lambda_{j}: j \in J\right\}$ is a $K$-g-frame for $\mathscr{U}$ with respect to $\left\{\mathscr{V}_{j}: j \in J\right\}$ with the frame bounds $A$ and $B$. Suppose that $T_{1}, T_{2} \in L(\mathscr{U})$ are surjective on $\mathscr{U}$ and satisfy $T_{i} K=K T_{i}, i=1,2$. If $\sqrt{A} /\left\|T_{1}^{\dagger}\right\|>\sqrt{B}\left\|T_{2}-T_{1}\right\|\left\|K^{\dagger}\right\|$, then $\left\{\Lambda_{j} T_{1}^{*}: j \in J\right\}$ and $\left\{\Lambda_{j} T_{2}^{*}: j \in J\right\}$ are $K$-woven in $R(K)$ with the frame bounds $\left(\sqrt{A} /\left\|T_{1}^{\dagger}\right\|-\sqrt{B}\left\|T_{2}-T_{1}\right\|\left\|K^{\dagger}\right\|\right)^{2}$ and $B\left(\left\|T_{1}\right\|^{2}+\left\|T_{2}\right\|^{2}\right)$.

In Ref. 12, the authors reported that, in general, applying two different operators to woven frames can give frames that are not woven (see Example 2 in Ref. 12). Corollary 3 also provides us with a sufficient condition for applying different operators $\left(T_{1}, T_{2}\right)$ to $\left\{\Lambda_{j}: j \in J\right\}$ such that $\left\{\Lambda_{j} T_{1}^{*}: j \in J\right\}$ and $\left\{\Lambda_{j} T_{2}^{*}: j \in J\right\}$ are woven in $\mathscr{U}$ (here, $K=I_{\mathscr{U}}$ and, clearly, $\left\{\Lambda_{j}: j \in J\right\}$ is woven with itself).

Note also that Corollary 3 is a generalization of Proposition 6.2 in Ref. 12. In fact, let $K=T_{1}=I_{\mathscr{U}}$; then $\left\{\Lambda_{j}: j \in J\right\}$ is a $g$-frame for $\mathscr{U}$ and $\sqrt{A} /\left\|T_{1}^{\dagger}\right\|>$ $\sqrt{B}\left\|T_{2}-T_{1}\right\|\left\|K^{\dagger}\right\|$ in Corollary 3 can be rewritten as $\left\|I_{\mathscr{U}}-T_{2}\right\|^{2}<A / B$. Then, from Corollary 3 , we can obtain a g-frame version of Proposition 6.2 in Ref. 12.

Corollary 4 Suppose that $\left\{\Lambda_{j}: j \in J\right\}$ is a g-frame for $\mathscr{U}$ with respect to $\left\{\mathscr{V}_{j}: j \in J\right\}$, with the frame bounds $A$ and $B$. Suppose that $T$ is surjective on $\mathscr{U}$. If $\left\|I_{\mathscr{U}}-T\right\|^{2}<A / B$, then $\left\{\Lambda_{j}: j \in J\right\}$ and $\left\{\Lambda_{j} T^{*}: j \in J\right\}$ are woven in $\mathscr{U}$ with the frame bounds $\left(\sqrt{A}-\sqrt{B}\left\|T-I_{\mathscr{U}}\right\|\right)^{2}$ and $B\left(1+\|T\|^{2}\right)$. 
Furthermore, if we let $\Lambda_{j} f=\left\langle f, f_{i}\right\rangle, \mathscr{V}_{j}=\mathbb{C}$, $j \in J$. Then $\left\{\Lambda_{j}: j \in J\right\}$ is a g-frame for $\mathscr{U}$ with respect to $\left\{\mathscr{V}_{j}: j \in J\right\}$ if and only if $\left\{f_{j}\right\}_{j \in J}$ is a frame for $\mathscr{U}$. Hence from Corollary 4 , we can obtain Proposition 6.2 in Ref. 12.

The next theorem tells us that, under condition (14), $\left\{\Lambda_{j}: j \in J\right\}$ and $\left\{\Gamma_{j}: j \in J\right\}$ are $K$-woven in $R(K)$, where $\left\{\Lambda_{j}: j \in J\right\}$ and $\left\{\Gamma_{j}: j \in J\right\}$ are assumed to be $K$-g-frames for $\mathscr{U}$.

Theorem 3 Let $K \in L(\mathscr{U})$ be an operator with a closed range. Suppose that $\left\{\Lambda_{j}: j \in J\right\}$ and $\left\{\Gamma_{j}: j \in J\right\}$ are $K$-g-frames for $\mathscr{U}$ with respect to $\left\{\mathscr{V}_{j}: j \in J\right\}$ with the frame bounds $A_{1}, B_{1}$ and $A_{2}, B_{2}$, respectively. If there exist $\alpha, \beta, \gamma \in[0, \infty)$ satisfying $A_{1}>\left(\sqrt{B_{1}}+\right.$ $\left.\sqrt{B_{2}}\right)\left(\alpha \sqrt{B_{1}}+\beta \sqrt{B_{2}}+\gamma\right)\left\|K^{\dagger}\right\|^{2}$ such that, for any $\left\{g_{j}\right\}_{j \in J} \in l^{2}\left(\left\{\mathscr{V}_{j}\right\}_{j \in J}\right)$,

$$
\begin{aligned}
&\left\|\sum_{j \in J}\left(\Lambda_{j}^{*}-\Gamma_{j}^{*}\right) g_{j}\right\| \leqslant \alpha\left\|\sum_{j \in J} \Lambda_{j}^{*} g_{j}\right\| \\
&+\beta\left\|\sum_{j \in J} \Gamma_{j}^{*} g_{j}\right\|+\gamma\left\|\left\{g_{j}\right\}_{j \in J}\right\|,
\end{aligned}
$$

then $\left\{\Lambda_{j}: j \in J\right\}$ and $\left\{\Gamma_{j}: j \in J\right\}$ are $K$-woven in $R(K)$ with the universal frame bounds $A_{1}-$ $\left(\sqrt{B_{1}}+\sqrt{B_{2}}\right)\left(\alpha \sqrt{B_{1}}+\beta \sqrt{B_{2}}+\gamma\right)\left\|K^{\dagger}\right\|^{2}$ and $B_{1}+$ $B_{2}$.

To prove Theorem 3, we need to give a lemma as follows.

Lemma 2 Let $\left\{\Lambda_{j}: j \in J\right\}$ and $\left\{\Gamma_{j}: j \in J\right\}$ be $K$ - $g$ frames for $\mathscr{U}$ with respect to $\left\{\mathscr{V}_{j}: j \in J\right\}$, with the Bessel bounds $B_{1}$ and $B_{2}$ and the synthesis operators $T_{1}$ and $T_{2}$, respectively. If there exist $\alpha, \beta, \gamma>0$ such that (14) holds, then, for any subset $\sigma \subset J$, we have

$$
\begin{aligned}
& \left\|\sum_{j \in \sigma} \Lambda_{j}^{*} \Lambda_{j} f-\sum_{j \in \sigma} \Gamma_{j}^{*} \Gamma_{j} f\right\| \leqslant \\
& \quad\left(\sqrt{B_{1}}+\sqrt{B_{2}}\right)\left(\alpha \sqrt{B_{1}}+\beta \sqrt{B_{2}}+\gamma\right)\|f\| .
\end{aligned}
$$

Proof: For any $\left\{g_{j}\right\}_{j \in J} \in l^{2}\left(\left\{\mathscr{V}_{j}\right\}_{j \in J}\right)$, from (14), we obtain

$$
\begin{aligned}
& \left\|\left(T_{1}-T_{2}\right)\left(\left\{g_{j}\right\}_{j \in J}\right)\right\|=\left\|\sum_{j \in J}\left(\Lambda_{j}^{*}-\Gamma_{j}^{*}\right) g_{j}\right\| \\
& \quad \leqslant \alpha\left\|\sum_{j \in J} \Lambda_{j}^{*} g_{j}\right\|+\beta\left\|\sum_{j \in J} \Gamma_{j}^{*} g_{j}\right\|+\gamma\left\|\left\{g_{j}\right\}_{j \in J}\right\| \\
& \quad=\alpha\left\|T_{1}\left(\left\{g_{j}\right\}_{j \in J}\right)\right\|+\beta\left\|T_{2}\left(\left\{g_{j}\right\}_{j \in J}\right)\right\|+\gamma\left\|\left\{g_{j}\right\}_{j \in J}\right\| \\
& \quad \leqslant \alpha\left\|T_{1}\right\|\left\|\left\{g_{j}\right\}_{j \in J}\right\|+\beta\left\|T_{2}\right\|\left\|\left\{g_{j}\right\}_{j \in J}\right\|+\gamma\left\|\left\{g_{j}\right\}_{j \in J}\right\| \\
& \quad \leqslant\left(\alpha \sqrt{B_{1}}+\beta \sqrt{B_{2}}+\gamma\right)\left\|\left\{g_{j}\right\}_{j \in J}\right\| .
\end{aligned}
$$

It follows that

$$
\left\|T_{1}-T_{2}\right\| \leqslant \alpha \sqrt{B_{1}}+\beta \sqrt{B_{2}}+\gamma
$$

since $\left\{g_{j}\right\}_{j \in J} \in l^{2}\left(\left\{\mathscr{V}_{j}\right\}_{j \in J}\right)$ is arbitrary.

Denote $\Phi_{j}=\Lambda_{j}-\Gamma_{j}, j \in J$, and the synthesis operator of $\left\{\Phi_{j}\right\}_{j \in J}$ by $T_{3}$. It is trivial to show that $\left\{\Phi_{j}\right\}_{j \in J}$ is a g-Bessel sequence and $T_{3}=T_{1}-T_{2}$. For any $\sigma \subset I, f \in \mathscr{H}$, we have

$$
\begin{aligned}
& \left\|\sum_{j \in \sigma} \Lambda_{j}^{*} \Lambda_{j} f-\sum_{j \in \sigma} \Gamma_{j}^{*} \Gamma_{j} f\right\| \\
& \quad=\left\|\sum_{j \in \sigma} \Lambda_{j}^{*} \Lambda_{j} f-\sum_{j \in \sigma} \Gamma_{j}^{*} \Lambda_{j} f+\sum_{j \in \sigma} \Gamma_{j}^{*} \Lambda_{j} f-\sum_{j \in \sigma} \Gamma_{j}^{*} \Gamma_{j} f\right\| \\
& \quad=\left\|\sum_{j \in \sigma}\left(\Lambda_{j}-\Gamma_{j}\right)^{*} \Lambda_{j} f+\sum_{j \in \sigma} \Gamma_{j}^{*}\left(\Lambda_{j}-\Gamma_{j}\right) f\right\| \\
& \quad \leqslant\left\|\sum_{j \in \sigma} \Phi_{j}^{*} \Lambda_{j} f\right\|+\left\|\sum_{j \in \sigma} \Gamma_{j}^{*} \Phi_{j} f\right\| \\
& \quad \leqslant\left\|T_{3}\right\|\left\|T_{1}\right\|\|f\|+\left\|T_{2}\right\|\left\|T_{3}\right\|\|f\| \\
& \quad=\left(\left\|T_{1}\right\|+\left\|T_{2}\right\|\right)\left\|T_{1}-T_{2}\right\|\|f\| \\
& \quad \leqslant\left(\sqrt{B_{1}}+\sqrt{B_{2}}\right)\left(\alpha \sqrt{B_{1}}+\beta \sqrt{B_{2}}+\gamma\right)\|f\|,
\end{aligned}
$$

where the last inequality is obtained by (16). Hence (15) holds.

Proof Theorem 3: For any $f \in R(K)$ and any partition $\left\{\sigma_{j}\right\}_{j=1}^{2}$ of $J$, we have

$$
\begin{aligned}
& \sum_{j \in \sigma_{1}}\left\|\Lambda_{j} f\right\|^{2}+\sum_{j \in \sigma_{2}}\left\|\Gamma_{j} f\right\|^{2} \\
& =\sum_{j \in J}\left\|\Lambda_{j} f\right\|^{2}+\sum_{j \in \sigma_{2}}\left\|\Gamma_{j} f\right\|^{2}-\sum_{j \in \sigma_{2}}\left\|\Lambda_{j} f\right\|^{2} \\
& \geqslant A_{1}\left\|K^{*} f\right\|^{2}-\left\langle\sum_{j \in \sigma_{2}} \Lambda_{j}^{*} \Lambda_{j} f-\sum_{j \in \sigma_{2}} \Gamma_{j}^{*} \Gamma_{j} f, f\right\rangle \\
& \geqslant A_{1}\left\|K^{*} f\right\|^{2}-\left\|\sum_{j \in \sigma_{2}} \Lambda_{j}^{*} \Lambda_{j} f-\sum_{j \in \sigma_{2}} \Gamma_{j}^{*} \Gamma_{j} f\right\|\|f\| \\
& \geqslant A_{1}\left\|K^{*} f\right\|^{2}-\left(\sqrt{B_{1}}+\sqrt{B_{2}}\right)\left(\alpha \sqrt{B_{1}}+\beta \sqrt{B_{2}}+\gamma\right)\|f\|^{2} \\
& \geqslant\left[A_{1}-\left(\sqrt{B_{1}}+\sqrt{B_{2}}\right)\left(\alpha \sqrt{B_{1}}+\beta \sqrt{B_{2}}+\gamma\right)\left\|K^{\dagger}\right\|^{2}\right]\left\|K^{*} f\right\|^{2},
\end{aligned}
$$

where the third and fourth inequalities are, respectively, deduced by (15) and (6). The upper bound for every weaving is trivial. Hence $\left\{\Lambda_{j}: j \in J\right\}$ and $\left\{\Gamma_{j}: j \in J\right\}$ are $K$-woven in $R(K)$.

In case $K=I_{\mathscr{U}}$ in Theorem 3, we can easily obtain a result as follows.

Corollary 5 Suppose that $\left\{\Lambda_{j}: j \in J\right\}$ and $\left\{\Gamma_{j}: j \in J\right\}$ are $g$-frames for $\mathscr{U}$ with respect to $\left\{\mathscr{V}_{j}: j \in J\right\}$, with the frame bounds $A_{1}, B_{1}$ and $A_{2}, B_{2}$, respectively. If there exist $\alpha, \beta, \gamma \in[0, \infty)$ satisfying $A_{1}>\left(\sqrt{B_{1}}+\right.$ $\left.\sqrt{B_{2}}\right)\left(\alpha \sqrt{B_{1}}+\beta \sqrt{B_{2}}+\gamma\right)$ such that, for any $\left\{g_{j}\right\}_{j \in J} \in$ 
$l^{2}\left(\left\{\mathscr{V}_{j}\right\}_{j \in J}\right)$

$$
\begin{aligned}
\left\|\sum_{j \in J}\left(\Lambda_{j}^{*}-\Gamma_{j}^{*}\right) g_{j}\right\| \leqslant \alpha \| & \sum_{j \in J} \Lambda_{j}^{*} g_{j} \| \\
& +\beta\left\|\sum_{j \in J} \Gamma_{j}^{*} g_{j}\right\|+\gamma\left\|\left\{g_{j}\right\}_{j \in J}\right\|,
\end{aligned}
$$

then $\left\{\Lambda_{j}: j \in J\right\}$ and $\left\{\Gamma_{j}: j \in J\right\}$ are woven in $\mathscr{U}$ with the universal frame bounds $A_{1}-$ $\left(\sqrt{B_{1}}+\sqrt{B_{2}}\right)\left(\alpha \sqrt{B_{1}}+\beta \sqrt{B_{2}}+\gamma\right)$ and $B_{1}+B_{2}$.

Furthermore, if $\alpha=\beta=0$ in Corollary 5, we can obtain a g-frame version of Theorem 6.1 in Ref. 12.

Next we provide the third type of perturbation condition (17) such that, under it, $\left\{\Lambda_{j}: j \in J\right\}$ and $\left\{\Gamma_{j}: j \in J\right\}$ can be $K$-woven in $R(K)$.

Theorem 4 Let $K \in L(\mathscr{U})$ be an operator with a closed range. Suppose that $\left\{\Lambda_{j}: j \in J\right\}$ and $\left\{\Gamma_{j}\right.$ : $j \in J\}$ are $K$-g-frames for $\mathscr{U}$ with respect to $\left\{\mathscr{V}_{j}\right.$ : $j \in J\}$ with the frame bounds $A_{1}, B_{1}$ and $A_{2}, B_{2}$, respectively. If there exist $\alpha, \beta, \gamma \in[0, \infty)$ satisfying $A_{1}>\left(\alpha B_{1}+\beta B_{2}+\gamma\right)\left\|K^{\dagger}\right\|^{2}$ such that, for any $\left\{g_{j}\right\}_{j \in J} \in l^{2}\left(\left\{\mathscr{V}_{j}\right\}_{j \in J}\right)$,

$$
\begin{array}{r}
\left\|\sum_{j \in \sigma} \Lambda_{j}^{*} \Lambda_{j} f-\sum_{j \in \sigma} \Gamma_{j}^{*} \Gamma_{j} f\right\| \leqslant \alpha\left\|\sum_{j \in \sigma} \Lambda_{j}^{*} \Lambda_{j} f\right\| \\
+\beta\left\|\sum_{j \in \sigma} \Gamma_{j}^{*} \Gamma_{j} f\right\|+\gamma\|f\|,
\end{array}
$$

then $\left\{\Lambda_{j}: j \in J\right\}$ and $\left\{\Gamma_{j}: j \in J\right\}$ are $K$-woven in $R(K)$ with the universal frame bounds $A_{1}-\left(\alpha B_{1}+\beta B_{2}+\right.$ $\gamma)\left\|K^{\dagger}\right\|^{2}$ and $B_{1}+B_{2}$.

Proof: Since $\left\{\Lambda_{j}: j \in J\right\}$ is a $K$-g-frame for $\mathscr{U}$ with the frame bounds $A_{1}$ and $B_{1}$, for any $\sigma \subset J$, we have

$$
\begin{aligned}
& \left\|\sum_{j \in \sigma} \Lambda_{j}^{*} \Lambda_{j} f\right\|=\sup _{g \in \mathscr{U},\|g\|=1}\left|\left\langle\sum_{j \in \sigma} \Lambda_{j}^{*} \Lambda_{j} f, g\right\rangle\right| \\
& =\sup _{g \in \mathscr{U},\|g\|=1}\left|\sum_{j \in \sigma}\left\langle\Lambda_{j} f, \Lambda_{j} g\right\rangle\right| \\
& \leqslant \sup _{g \in \mathscr{U},\|g\|=1} \sum_{j \in \sigma}\left|\left\langle\Lambda_{j} f, \Lambda_{j} g\right\rangle\right| \\
& \leqslant \sup _{g \in \mathscr{U},\|g\|=1}\left(\sum_{j \in J}\left\|\Lambda_{j} f\right\|^{2}\right)^{1 / 2}\left(\sum_{j \in J}\left\|\Lambda_{j} g\right\|^{2}\right)^{1 / 2} \\
& \leqslant \sup _{g \in \mathscr{U},\|g\|=1} \sqrt{B_{1}}\|f\| \sqrt{B_{1}}\|g\|=B_{1}\|f\| . \quad
\end{aligned}
$$

Similarly, we can obtain $\left\|\sum_{j \in \sigma} \Gamma_{j}^{*} \Gamma_{j} f\right\| \leqslant B_{2}\|f\|$.
Hence if we combine (17) and (18), it follows that

$$
\begin{aligned}
& \left\|\sum_{j \in \sigma} \Lambda_{j}^{*} \Lambda_{j} f-\sum_{j \in \sigma} \Gamma_{j}^{*} \Gamma_{j} f\right\| \\
& \quad \leqslant \alpha\left\|\sum_{j \in \sigma} \Lambda_{j}^{*} \Lambda_{j} f\right\|+\beta\left\|\sum_{j \in \sigma} \Gamma_{j}^{*} \Gamma_{j} f\right\|+\gamma\|f\| \\
& \quad \leqslant\left(\alpha B_{1}+\beta B_{2}+\gamma\right)\|f\| .
\end{aligned}
$$

For any $f \in R(K)$, and any partition $\left\{\sigma_{j}\right\}_{j=1}^{2}$ of $J$, by the same method of Theorem 3, we obtain

$$
\begin{aligned}
\sum_{j \in \sigma_{1}}\left\|\Lambda_{j} f\right\|^{2}+\sum_{j \in \sigma_{2}}\left\|\Gamma_{j} f\right\|^{2} \\
\quad \geqslant A_{1}\left\|K^{*} f\right\|^{2}-\left\|\sum_{j \in \sigma_{2}} \Lambda_{j}^{*} \Lambda_{j} f-\sum_{j \in \sigma_{2}} \Gamma_{j}^{*} \Gamma_{j} f\right\|\|f\| \\
\geqslant A_{1}\left\|K^{*} f\right\|^{2}-\left(\alpha B_{1}+\beta B_{2}+\gamma\right)\|f\|^{2} \\
\geqslant A_{1}\left\|K^{*} f\right\|^{2}-\left(\alpha B_{1}+\beta B_{2}+\gamma\right)\left\|K^{\dagger}\right\|^{2}\left\|K^{*} f\right\|^{2} \\
=\left(A_{1}-\left(\alpha B_{1}+\beta B_{2}+\gamma\right)\left\|K^{\dagger}\right\|^{2}\right)\left\|K^{*} f\right\|^{2},
\end{aligned}
$$

where the second inequality is deduced by (19). Hence $\left\{\Lambda_{j}: j \in J\right\}$ and $\left\{\Gamma_{j}: j \in J\right\}$ are $K$-woven in $R(K)$.

If $K=I_{\mathscr{U}}$, a result follows immediately from Theorem 4.

Corollary 6 Suppose that $\left\{\Lambda_{j}: j \in J\right\}$ and $\left\{\Gamma_{j}: j \in J\right\}$ are $g$-frames for $\mathscr{U}$ with respect to $\left\{\mathscr{V}_{j}: j \in J\right\}$ with the frame bounds $A_{1}, B_{1}$ and $A_{2}, B_{2}$, respectively. If there exist $\alpha, \beta, \gamma \in[0, \infty)$ satisfying $A_{1}>\alpha B_{1}+\beta B_{2}+\gamma$ such that, for any $\left\{g_{j}\right\}_{j \in J} \in l^{2}\left(\left\{\mathscr{V}_{j}\right\}_{j \in J}\right)$,

$$
\begin{aligned}
\| \sum_{j \in \sigma} \Lambda_{j}^{*} \Lambda_{j} f & -\sum_{j \in \sigma} \Gamma_{j}^{*} \Gamma_{j} f \| \\
& \leqslant \alpha\left\|\sum_{j \in \sigma} \Lambda_{j}^{*} \Lambda_{j} f\right\|+\beta\left\|\sum_{j \in \sigma} \Gamma_{j}^{*} \Gamma_{j} f\right\|+\gamma\|f\|,
\end{aligned}
$$

then $\left\{\Lambda_{j}: j \in J\right\}$ and $\left\{\Gamma_{j}: j \in J\right\}$ are woven in $\mathscr{U}$ with the universal frame bounds $A_{1}-\left(\alpha B_{1}+\beta B_{2}+\gamma\right)$ and $B_{1}+B_{2}$.

Acknowledgements: This work is partly supported by the Natural Science Foundation of Fujian Province, China (grant No. 2016J01014), and by the projects of Xiamen University of Technology (grant No. G2017005).

\section{REFERENCES}

1. Sun W (2006) G-frames and g-Riesz bases. $J$ Math Anal Appl 322, 437-452.

2. Xiao XC, Zhu YC, Shu ZB, Ding ML (2015) G-frames with bounded linear operators. Rocky Mountain $J$ Math 45, 675-693. 
3. Guo XX (2015) New characterizations of g-Bessel sequences and g-Riesz bases in Hilbert spaces. Results Math 68, 361-374.

4. Khosravi A, Azandaryani MM (2012) Fusion frames and g-frames in tensor product and direct sum of Hilbert spaces. Appl Anal Discrete Math 6, 287-303.

5. Khosravi A, Azandaryani MM (2013) G-frames and direct sums. Bull Malays Math Sci Soc 36, 313-323.

6. Găvruţa L (2012) Frames for operators. Appl Comp Harm Anal 32, 139-144.

7. Xiang ZQ, Li YM (2016) Frame sequences and dual frames for operators. ScienceAsia 42, 222-230.

8. Xiao XC, Zhu YC, Găvruţa L (2013) Some properties of $K$-frames in Hilbert spaces. Results Math 63, 1243-1255.

9. Guo XX (2016) Canonical dual $K$-Bessel sequences and dual $K$-Bessel generators for unitary systems of Hilbert spaces. J Math Anal Appl 444, 598-609.

10. Khosravi A, Azandaryani MM (2014) Approximate duality of g-frames in Hilbert spaces. Acta Math Sci 34, 639-652.

11. Xiao XC, Zhu YC (2017) Exact $K$-g-frames in Hilbert spaces. Results Math 72, 1329-1339.

12. Bemrose T, Casazza PG, Grochenig K, Lammers MC,
Lynch RG (2016) Weaving frames. Oper Matrices 10, 1093-1116.

13. Casazza PG, Freeman D, Lynch RG (2016) Weaving Schauder frames. $J$ Approx Theory 211, 42-60.

14. Casazza PG, Lynch RG (2015) Weaving properties of Hilbert space frames. In: International Conference on Sampling Theory and Applications, pp 110-114.

15. Deepshikha, Agarwal S, Vashisht LK, Verma G (2017) On weaving fusion frames for Hilbert spaces. In: International Conference on Sampling Theory and Applications, pp 381-385.

16. Khosravi A, Banyarani JS (2018) Weaving g-frames and weaving fusion frames. Bull Malays Math Sci Soc, $1-19$.

17. Vashisht LK, Agarwal S, Deepshikha (2018) On generalized weaving frames in Hilbert spaces. Rocky Mountain J Math 48, 661-685.

18. Vashisht LK, Deepshikha (2016) Weaving properties of generalized continuous frames generated by an iterated function system. J Geom Phys 110, 282-295.

19. Deepshikha, Vashisht LK (2018) Weaving K-frames in Hilbert spaces. Results Math 73, 81.

20. Christensen O (2003) An Introduction to Frames and Riesz Bases, Birkhäuser, Boston. 